\title{
Individual Differences in Embodied Distance Estimation in Virtual Reality
}

\author{
Mar Gonzalez-Franco* \\ Microsoft Research
}

\author{
Parastoo Abtahi \\ Stanford University
}

\author{
Anthony Steed \\ Microsoft Research \\ University College London
}

\begin{abstract}
There are important individual differences when experiencing VR setups. We ran a study with 20 participants who got a scale-matched avatar and were asked to blind-walk to a VR target placed 2.5 meters away. In such setups, people typically underestimate distances by approximately $10 \%$ when virtual environments are viewed through head mounted displays. Consistent with previous studies we found that the underestimation was significantly reduced the more embodied the participants were. However, not all participants developed the same level of embodiment when exposed to the exact same conditions.
\end{abstract}

\section{INTRODUCTION}

Self-avatar representation within the virtual environment is known to have a wide variety of impacts on the user [21, 4, 2, 22]. For example, having a body, and more importantly, being encouraged to use it has a positive impact on reports of presence in virtual environments [20]. In a well-known study on locomotion, Usoh et al. compared real-walking to walking on the spot to flying with a joystick [24] and found that the subjective experience was significantly correlated with a sense of ownership over the virtual body. Subsequently, the sense of ownership of a virtual body has been extensively studied [9].

When it comes to locomotion, it has been shown that the presence of a self-avatar changes stepping behavior. In particular, if the feet are tracked, the self-avatar can be properly articulated in its gait. Lin et al. showed that the self-avatar animated by foot tracking improved estimations of heights for stepping over and under obstacles [12]. Similarly, Kim et al. found that the presence of representations of the feet would lead to the users placing their feet more carefully [10].

In this paper we further study the impact of embodied avatars during a distance estimation task inside VR [1].

The ability to accurately convey distances through virtual environments is key to many potential applications: from veridical transfer of training through to ensuring fairness in competitive games. As immersive VR is almost always experienced from a first-person, egocentric point of view, it already benefits from many cues that aid distance estimation: the eye-height can be naturally set at the correct height and the user can make natural gestures. However, whenever studied, it is found that users of virtual environments tend to underestimate distances, and thus when asked to walk, they walk slightly shorter (e.g. see [14, 6, 23, 18]).

Aside from the raw compression of distance, recently, Janeh et al. showed that a side-effect of motion compression seems to be changes in gait [8]. Gait is thus one aspect of behaviour that we will study in our experiment.

Prior work on embodiment has found that ownership over a self-avatar improves distance estimation, though the compression is not completely removed [15, 19] 17]. However, control of the

*e-mail: margon@microsoft.com embodiment is important as the body is not just providing a measure of scale for the avatar but also a way to interact with the avatar [16, 11].

Our focus is to better understand the individual differences of users by analyzing their actual walking behaviour.

\section{Materials and Methods}

Twenty participants ( 5 female, ages $25-55, \mu=34$ ) with normal stereo vision participated in our experiment and received a gift card as compensation.They wore a HTC Vive HMD, with hand-held controllers and trackers attached to their shoes. We used the Root Motion full-body inverse kinematics library to animate a Dummy avatar. They were tracked with lighthouses and a TPCAST Wireless Adapter to enable an untethered VR experience in our $2.8 m \times 4 m \times 2.8 m$ study room. To guide the participants along a path in the room, we rendered a green $\mathrm{X}$ mark on the ground and a capsule positioned at their eye height, as shown in figure 1

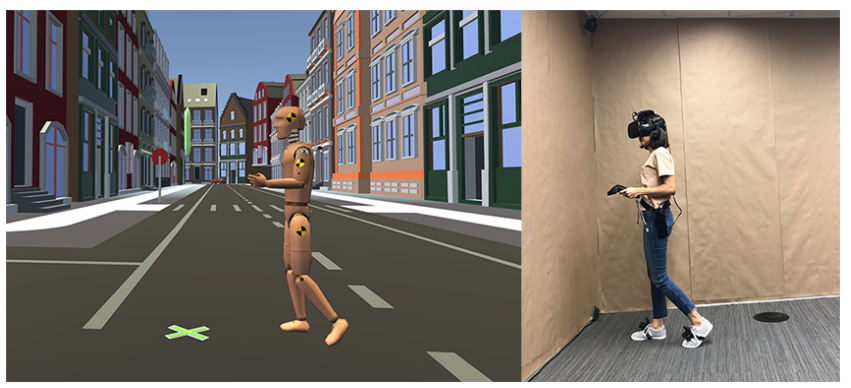

Figure 1: Participant walking towards a target before the distance estimation task. Left) The avatar and the green target in the virtual city. Right) Participant holding the controllers while wearing the battery pack, wireless HMD, and foot trackers.

The experiment followed this procedure:

- 10 seconds of walking towards green targets. Once the target is reached, the next target's position is randomly generated within the physical room, providing the participant with a walkable path in the virtual city.

- After 10 seconds upon arrival to the target, the scene disappears and is replaced by a white plane. A red target then appears at a prespecified distance from the Participant.

- After 5 seconds, the red target disappears and the participant walks in the empty scene to stop exactly where the red target was, and pull the trigger on the right-hand controller.

- The virtual scene reappears and a new trial starts.

The above sequence was repeated six times and the red target, positioned $2.5 \mathrm{~m}$ away from the participant. At the end of the experiment participants completed an embodiment questionnaire and the Nasa TLX questionnaire [5] 3]. 


\section{Embodiment}
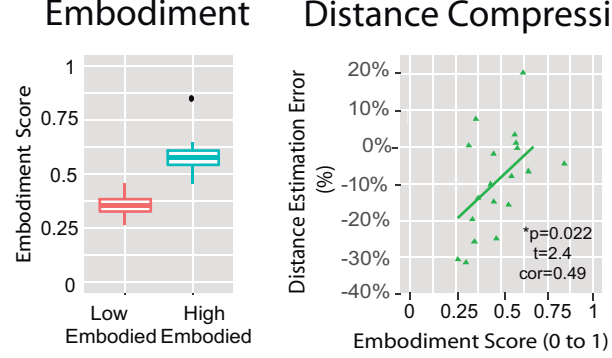

Individual Strides

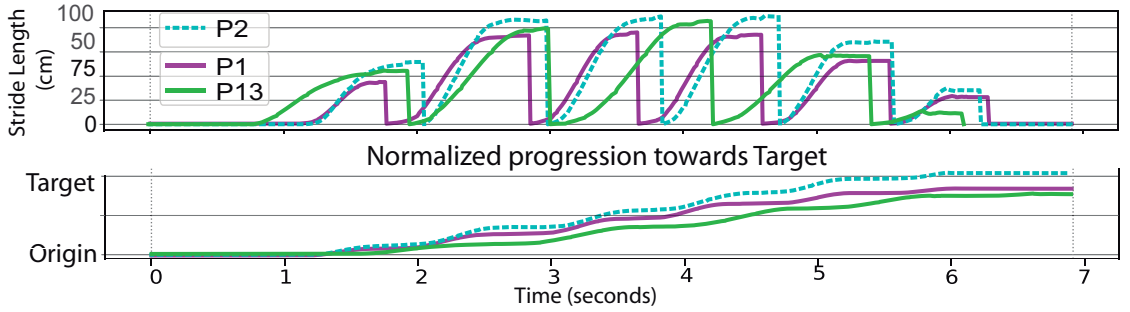

Figure 2: Walking behaviour changes for different avatar embodiment levels. For the individual strides we pick three participants (P2, high embodied, and P2,P13 low embodied) to be representatives because their cadence in steps is very comparable. Two main trends were identified for low embodied participants the reduction of stride length $(\mathrm{P} 1)$ or the reduction in the number of steps with a similar stride length (P13). In both cases the result was a greater distance compression for low embodied individuals.

\section{RESULTS}

\subsection{Embodiment Analysis}

Using Principal Component Analysis (PCA) we calculated the embodiment score of our participants [3] (Table 1). The average embodiment score (normalized from 0 to 1 ) in our experiment was $0.47 \pm 0.14$ SD. Participants could be clustered into high embodied $(0.59 \pm 0.11 \mathrm{SD}$ score $)$ and low embodied $(0.36 \pm 0.06 \mathrm{SD}$ score $)$; the distributions in the two groups differed significantly (MannWhitney $\mathrm{U}=0, \mathrm{n} 1=\mathrm{n} 2=10, p<0.0001$ ).

Table 1: PCA Factor Loadings

\begin{tabular}{l|l|l}
\hline Question & $\begin{array}{l}\text { Factor 1 } \\
\text { Embodied }\end{array}$ & $\begin{array}{l}\text { Factor 2 } \\
\text { Task }\end{array}$ \\
\hline $\begin{array}{l}\text { I felt as if the virtual body was my body. } \\
\text { It felt as if the virtual body I saw was }\end{array}$ & $\mathbf{0 . 6 6 2}$ & 0.174 \\
someone else. & & -0.304 \\
$\begin{array}{l}\text { The movements of the virtual body were } \\
\text { caused by my movement. }\end{array}$ & 0.400 & \\
$\begin{array}{l}\text { I felt like I could control the virtual body } \\
\text { as if it was my own body. }\end{array}$ & $\mathbf{0 . 6 0 6}$ & -0.119 \\
$\begin{array}{l}\text { I felt as if the virtual body was moving by } \\
\text { itself. }\end{array}$ & -0.326 & \\
$\begin{array}{l}\text { I felt as if the movements of the virtual body } \\
\text { were influencing my own movements. }\end{array}$ & $\mathbf{0 . 5 8 4}$ & \\
$\begin{array}{l}\text { I felt as if my (real) body was turning into } \\
\text { an avatar body. }\end{array}$ & $\mathbf{0 . 6 5 9}$ & -0.168 \\
$\begin{array}{l}\text { At some point it felt as if my real body was } \\
\text { starting to take on the posture or shape of the } \\
\text { virtual body that I saw. }\end{array}$ & $\mathbf{0 . 7 2 9}$ & 0.110 \\
$\begin{array}{l}\text { The task was mentally demanding. } \\
\text { The task was physically demanding. } \\
\text { The pace of the task felt hurried or rushed. }\end{array}$ & 0.196 & $\mathbf{0 . 7 8 4}$ \\
$\begin{array}{l}\text { I was successful at accomplishing what I was } \\
\text { asked to do. }\end{array}$ & -0.176 & $\mathbf{0 . 7 6 1}$ \\
$\begin{array}{l}\text { I had to work hard to accomplish what I was } \\
\text { asked to do. }\end{array}$ & 0.350 & -0.269 \\
$\begin{array}{l}\text { I felt insecure, discouraged, irritated, stressed, } \\
\text { and annoyed. }\end{array}$ & -0.280 & $\mathbf{0 . 5 7 5}$ \\
\hline
\end{tabular}

Note: question loading with high influence on the component are in bold.

\subsection{Walking Behaviour}

The distance error across all targets was $11 \pm 0.54 \%$, consistent with previous findings in which people underestimated distances in VR by $10 \%$ [7, [23]. We found a correlation between embodiment score and error for targets at more than $2 \mathrm{~m}$ (Pearson, cor $=0.49, \mathrm{t}=2.4, \mathrm{df}=18, \mathrm{p}=0.025)$. High embodied participants significantly reduced their error to $5 \%$ while participants with lower embodiment maintained estimation errors over $10 \%$.

We analyzed the walking behavior to better understand the improved estimation of distances for highly embodied participants. We found that low embodied participants stride length was $0.6 \pm 0.17 \mathrm{SD}$ meters and the high embodied participants stride length was $0.71 \pm 0.1$ SD meters (Welch test, $t=-2.1, d f=12.6, p=0.05)$. The stride length was adjusted by the participant's height and then normalized to the equivalent of a $1.7 \mathrm{~m}$ tall person. We observed two main trends, (see Figure 2). The average number of steps across conditions was $5.28 \pm 1.57$ for the low embodied, and $4.97 \pm 1.39$ for the high embodied $(p>0.4)$. Neither the speed nor the cadence of the strides were affected by the embodiment level.

\section{Discussion}

Given the walking behavioural results, we hypothesize that most low embodied individuals were actually compressing the distance to the target as a result of their reduced stride length. This stride compression effect was consistent for the majority of the low embodied participants, affecting 7 out of 10 participants. The rest of the low embodied participants (3 out of 10) did not show shorter strides, but rather a reduced number of steps that also translated into a distance estimation compression.

These results, together with previous findings, have significant implications for developers. For example, in collaborative VR, social clues are very important and users rely on implicit techniques such as proxemics or more directly distance perceptions to interact [13]. Our work highlights that providing a first person avatar might not be sufficient if the participants do not feel embodied. We believe that to achieve highly effective VR applications, individual perceptual differences need to be considered, as they ultimately translate into different behaviours in the virtual environment, as shown by the gait analysis.

We hypothesize that the behavioral alterations stemming from individual differences will have significant impact on other higherlevel tasks such as navigation or social group forming.

\section{References}

[1] P. Abtahi, M. Gonzalez-Franco, E. Ofek, and A. Steed. Im a giant: Walking in large virtual environments at high speed gains. In Proceedings of the 2019 CHI Conference on Human Factors in Computing Systems. ACM, 2019.

[2] M. Gonzalez-Franco and J. Lanier. Model of illusions and virtual reality. Frontiers in psychology, 8:1125, 2017.

[3] M. Gonzalez-Franco and T. C. Peck. Avatar embodiment. towards a standardized questionnaire. Frontiers in Robotics and AI, 5:74, 2018. 
[4] M. Gonzalez-Franco, D. Perez-Marcos, B. Spanlang, and M. Slater. The contribution of real-time mirror reflections of motor actions on virtual body ownership in an immersive virtual environment. In Virtual Reality Conference (VR), 2010 IEEE, pages 111-114. IEEE, 2010.

[5] S. G. Hart and L. E. Staveland. Development of nasa-tlx (task load index): Results of empirical and theoretical research. In Advances in psychology, volume 52, pages 139-183. Elsevier, 1988.

[6] V. Interrante, B. Ries, and L. Anderson. Distance Perception in Immersive Virtual Environments, Revisited. In IEEE Virtual Reality Conference (VR 2006), pages 3-10, Mar. 2006.

[7] V. Interrante, B. Ries, J. Lindquist, M. Kaeding, and L. Anderson. Elucidating factors that can facilitate veridical spatial perception in immersive virtual environments. Presence: Teleoperators and Virtual Environments, 17(2):176-198, 2008.

[8] O. Janeh, E. Langbehn, F. Steinicke, G. Bruder, A. Gulberti, and M. Poetter-Nerger. Walking in Virtual Reality: Effects of Manipulated Visual Self-Motion on Walking Biomechanics. ACM Trans. Appl. Percept., 14(2):12:1-12:15, Jan. 2017.

[9] K. Kilteni, A. Maselli, K. Koerding, and M. Slater. Over my fake body: body ownership illusions for studying the multisensory basis of own-body perception. Frontiers in Human Neuroscience, 9(141), 2015 .

[10] A. Kim, K. S. Kretch, Z. Zhou, and J. M. Finley. The quality of visual information about the lower extremities influences visuomotor coordination during virtual obstacle negotiation. Journal of Neurophysiology, 120(2):839-847, Aug. 2018.

[11] M. Leyrer, S. A. Linkenauger, H. H. Blthoff, U. Kloos, and B. Mohler. The Influence of Eye Height and Avatars on Egocentric Distance Estimates in Immersive Virtual Environments. In Proceedings of the ACM SIGGRAPH Symposium on Applied Perception in Graphics and Visualization, APGV'11, pages 67-74, New York, NY, USA, 2011. ACM.

[12] Q. Lin, J. Rieser, and B. Bodenheimer. Stepping over and Ducking Under: The Influence of an Avatar on Locomotion in an HMD-based Immersive Virtual Environment. In Proceedings of the ACM Symposium on Applied Perception, SAP '12, pages 7-10, New York, NY, USA, 2012. ACM.

[13] J. Llobera, B. Spanlang, G. Ruffini, and M. Slater. Proxemics with multiple dynamic characters in an immersive virtual environment. ACM Transactions on Applied Perception (TAP), 8(1):3, 2010.

[14] J. M. Loomis, J. M. Knapp, et al. Visual perception of egocentric distance in real and virtual environments. Virtual and adaptive environments, pages 21-46, 2003.

[15] B. J. Mohler, H. H. Blthoff, W. B. Thompson, and S. H. CreemRegehr. A full-body avatar improves egocentric distance judgments in an immersive virtual environment. In Proceedings of the 5th symposium on Applied perception in graphics and visualization, page 194 ACM, 2008.

[16] B. J. Mohler, S. H. Creem-Regehr, W. B. Thompson, and H. H. Blthoff. The Effect of Viewing a Self-Avatar on Distance Judgments in an HMD-Based Virtual Environment. Presence: Teleoperators and Virtual Environments, 19(3):230-242, June 2010.

[17] L. Phillips, B. Ries, M. Kaedi, and V. Interrante. Avatar self-embodiment enhances distance perception accuracy in nonphotorealistic immersive virtual environments. In 2010 IEEE Virtual Reality Conference (VR), pages 115-1148, Boston, MA, USA, Mar. 2010. IEEE.

[18] R. S. Renner, B. M. Velichkovsky, and J. R. Helmert. The perception of egocentric distances in virtual environments - A review. ACM Computing Surveys, 46(2):1-40, Nov. 2013.

[19] B. Ries, V. Interrante, M. Kaeding, and L. Anderson. The Effect of Self-embodiment on Distance Perception in Immersive Virtual Environments. In Proceedings of the 2008 ACM Symposium on Virtual Reality Software and Technology, VRST '08, pages 167-170, New York, NY, USA, 2008. ACM.

[20] M. Slater, J. McCarthy, and F. Maringelli. The Influence of Body Movement on Subjective Presence in Virtual Environments. Human Factors, 40(3):469-477, Sept. 1998.

[21] B. Spanlang, J.-M. Normand, D. Borland, K. Kilteni, E. Giannopoulos, A. Poms, M. Gonzlez-Franco, D. Perez-Marcos, J. Arroyo-
Palacios, X. N. Muncunill, and M. Slater. How to build an embodiment lab: Achieving body representation illusions in virtual reality. Frontiers in Robotics and AI, 1:9, 2014.

[22] A. Steed, Y. Pan, F. Zisch, and W. Steptoe. The impact of a self-avatar on cognitive load in immersive virtual reality. In 2016 IEEE Virtual Reality (VR), pages 67-76, Greenville, SC, USA, Mar. 2016. IEEE.

[23] W. B. Thompson, J. E. Swan, D. Proffitt, J. K. Kearney, and V. Interrante. Elucidating Factors that can Facilitate Veridical Spatial Perception in Immersive Virtual Environments. In 2007 IEEE Virtual Reality Conference, pages 11-18, Mar. 2007.

[24] M. Usoh, K. Arthur, M. C. Whitton, R. Bastos, A. Steed, M. Slater, and F. P. Brooks. Walking $>$ walking-in-place $>$ flying, in virtual environments. In Proceedings of the 26th annual conference on Computer graphics and interactive techniques - SIGGRAPH' '99, pages 359-364, Not Known, 1999. ACM Press. 